\title{
Avaliação da adequação da ingestão de nutrientes na prática clínica
}

\author{
Assessment of nutrient intake adequacy \\ in clinical practice
}

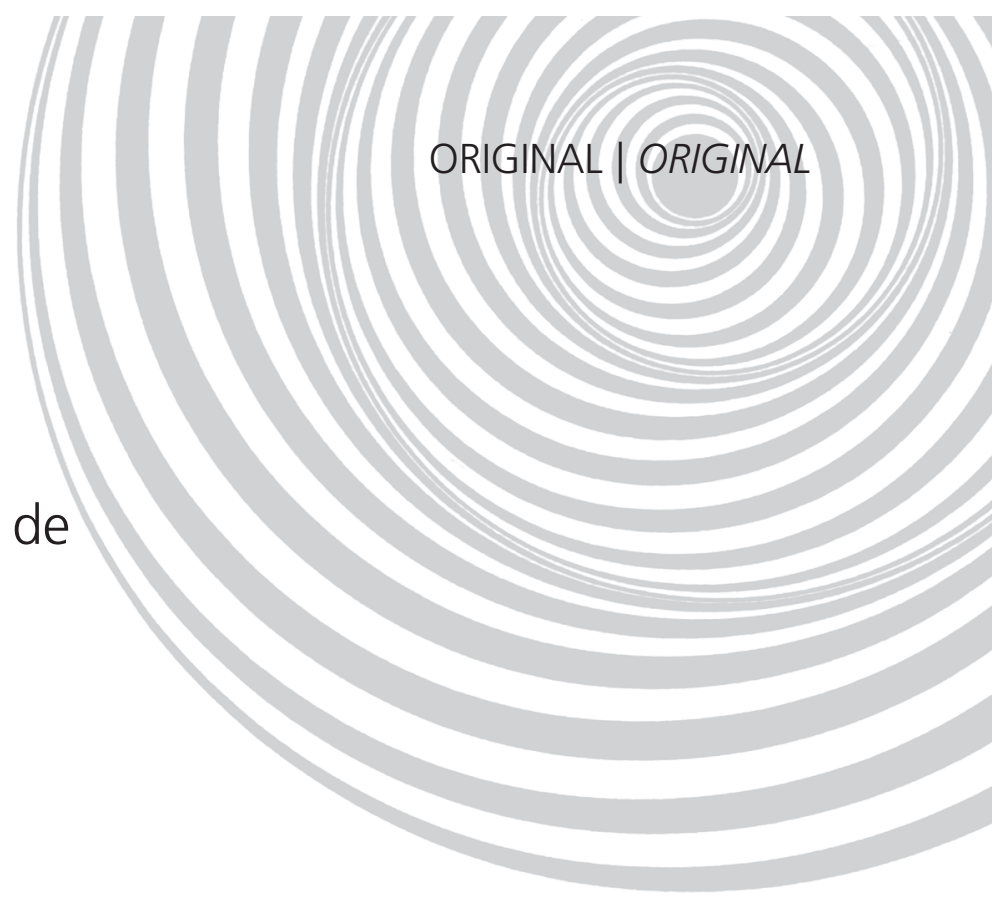

Dirce Maria Lobo MARCHIONI ${ }^{1}$

Eliseu VERLY JUNIOR ${ }^{1}$

Chester Luis Galvão CESAR²

Regina Mara FISBERG ${ }^{1}$

RE S U M O

\section{Objetivo}

Para se avaliar a adequação da ingestão de nutrientes, é necessário determinar qual é a quantidade necessária para as funções normais do indivíduo, bem como a sua ingestão habitual. A necessidade de nutrientes varia de indivíduo para indivíduo, assumindo um coeficiente de variação de 10\% a 15\%. Além disso, a ingestão individual está sujeita à variação de consumo no dia a dia, conhecida como variabilidade intrapessoal. Considerando que as informações sobre a variação intrapessoal disponíveis na literatura são as da população norte-americana, este estudo tem dois objetivos: (1) disponibilizar valores de desvio-padrão intrapessoal de ingestão de nutrientes estimados em população brasileira, que possam ser aplicados no cálculo da adequação da ingestão de nutrientes; e (2) oferecer valores teóricos que apoiem a tomada de decisão, na prática clínica, quanto à adequação da ingestão de nutrientes selecionados por um indivíduo, calculados com base nos desvios-padrão encontrados e com três níveis de confiabilidade.

\section{Métodos}

Foram utilizados dados de adolescentes, adultos e idosos que participaram do inquérito de saúde de base populacional no município de São Paulo (Inquérito de Saúde de São Paulo). Foram entrevistados 865 indivíduos, que responderam a um recordatório de 24 horas, replicados em uma subamostra de 294 sujeitos. Esses dados foram utilizados para o cálculo do desvio-padrão por meio de modelos de efeitos aleatórios.

\section{Resultados}

Os coeficientes estimados variaram de $40 \%$ para o zinco, a $127 \%$ para a vitamina C.

\section{Conclusão}

Os desvios-padrão encontrados podem ser utilizados na avaliação quantitativa da adequação da ingestão de vitamina E, B6, tiamina, riboflavina, cálcio, magnésio, ferro, zinco, sódio, folato e niacina, propiciando mais um elemento formativo do diagnóstico do estado nutricional.

Termos de indexação: Avaliação nutricional. Ingestão de alimentos. Necessidades nutricionais. Nutrientes.

1 Universidade de São Paulo, Faculdade de Saúde Pública, Departamento de Nutrição. Av. Dr. Arnaldo, 715, Cerqueira César, 01246-904, São Paulo, SP, Brasil. Correspondência para/Correspondence to: D.M.L. MARCHIONI. E-mail: <marchioni@usp.br>.

${ }^{2}$ Universidade de São Paulo, Faculdade de Saúde Pública, Departamento de Epidemiologia. São Paulo, SP, Brasil. 
826 | D.M.L. MARCHIONI et al.

\section{A B S T R A C T}

\section{Objective}

Quantitative assessment of nutrient intake requires knowing how much of the nutrient is needed for the normal functions of the individual and his or her habitual intake. Nutrient requirements vary between individuals; the assumed coefficient of variation is 10 to $15 \%$. Individual intake is subject to variation of daily intake, which is known as intrapersonal variability. Currently, information on intrapersonal variation in the literature refers to the American population. This study has two objectives: 1) determine the intrapersonal standard deviation of nutrient intake for the Brazilian population that can be used for calculating the adequacy of nutrient intake; and 2) offer theoretical values that support decision making in clinical practice regarding the adequacy of nutrient intake selected by an individual, based on standard deviations and with three levels of reliability.

\section{Methods}

Data from teenagers, adults and elderly individuals who participated in the population-based health survey done in the city of São Paulo were used. These individuals $(n=865)$ answered a 24-hour recall, replicated in a subsample of 294 individuals. These data were used for calculating the standard deviation using random effects models.

\section{Results}

The estimated coefficients varied from $40 \%$ for zinc to $127 \%$ for vitamin C.

\section{Conclusion}

The standard deviations can be used for the assessment of quantitative intake adequacies of vitamin E, pyridoxine, thiamin, riboflavin, calcium, magnesium, iron, zinc, folate and niacin. Hence, one more element for nutritional status diagnosis is made available for clinical assessment.

Indexing terms: Nutrition assessment. Eating. Nutritional requirements. Nutrients.

\section{N T R O D U Ç Ã O}

A avaliação nutricional busca detectar problemas nutricionais, colaborando para a promoção e a recuperação da saúde. Na prática clínica, a análise da história clínica, dietética e social, bem como os dados antropométricos, bioquímicos e a interação entre drogas e nutrientes, são utilizados para estabelecer o diagnóstico nutricional e servir de base para o planejamento e a orientação dietética. A avaliação da ingestão de nutrientes é parte da avaliação nutricional, sendo utilizada para a tomada de decisão quanto à adequação do consumo alimentar do indivíduo e para o estabelecimento da conduta dietoterápica, em conjunto com os outros parâmetros citados ${ }^{1}$.

A avaliação quantitativa da ingestão de nutrientes requer a informação, tanto da necessidade de nutriente para as funções normais do indivíduo, quanto de sua ingestão habitual. Ressalta-se que a necessidade de nutrientes varia de indivíduo para indivíduo, segundo seu estágio de vida, estado fisiológico, atividade física, características metabólicas e outras características pessoais. A ingestão habitual, por sua vez, é definida como a média de ingestão individual durante um longo período de tempo². Para sua obtenção, em geral, é necessário um número elevado de dias de aplicação de inquéritos dietéticos, quando são utilizados métodos que refletem acuradamente o consumo de curto prazo, como o registro alimentar ou o recordatório de 24 horas $^{3}$. Nesse sentido, devido à variabilidade que naturalmente ocorre na ingestão de alimentos no dia a dia (variabilidade intrapessoal), a estimativa do consumo baseada em poucos dias de coleta, ao falhar na captação dessa flutuação, pode conduzir a conclusões incorretas sobre a adequação da ingestão de um dado nutriente por um determinado indivíduo 4 .

O método para avaliação da ingestão de nutrientes em indivíduos, proposto pelo Instituto de Medicina (IOM) ${ }^{5}$, órgão que publicou as recomendações para ingestão de nutrientes (Dietary 
Reference Intakes - DRI) leva em consideração tanto a variabilidade da necessidade de nutrientes nos indivíduos quanto a variabilidade intrapessoal da ingestão. Para sua aplicação, entretanto, é preciso utilizar valores de variabilidade intrapessoal, expressos pelo desvio-padrão intrapessoal de ingestão de cada nutriente, obtidos em estudos com a mesma população. No entanto, nos estudos desenvolvidos no Brasil, há uma lacuna, pois atualmente apenas os valores de desvio-padrão intrapessoal obtidos na população norte-americana estão disponíveis para serem usados nas equações propostas. Considerando a influência de fatores culturais e econômicos no consumo de alimentos, infere-se que o emprego de parâmetros estrangeiros na avaliação da adequação de nutrientes em indivíduos brasileiros pode ser um potencial viés ${ }^{6}$. Assim, o presente estudo tem como objetivos: (1) disponibilizar valores de desvio-padrão intrapessoal de ingestão de nutrientes que possam ser aplicados no cálculo da adequação da ingestão de nutrientes em indivíduos brasileiros; e (2) oferecer valores teóricos que apoiem a tomada de decisão, na prática clinica, quanto à adequação da ingestão de nutrientes selecionados por um indivíduo, calculados com base nos desvios-padrão encontrados e com três níveis de confiabilidade.

\section{M É T O D O S}

Os cálculos para estimativa da variabilidade de ingestão dos nutrientes selecionados foram baseados na amostra composta por indivíduos que participaram do estudo "Inquérito de Saúde de São Paulo" (ISA-SP). Trata-se de estudo transversal com amostra representativa da população do município, cujos detalhes podem ser obtidos em publicação anterior?

Para o presente estudo, foram sorteados 1235 indivíduos, dos quais se obtiveram 865 entrevistas (273 adolescentes, 301 adultos e 291 idosos). Os indivíduos foram entrevistados em seus domicílios, por entrevistadores previamente treinados. O consumo alimentar foi coletado pelo método Recordatório de 24 horas (R24h). Para o cálculo do desvio-padrão intrapessoal da ingestão de nutrientes, é necessária uma segunda aplicação do R24h em pelo menos uma subamostra representativa da população de estudo ${ }^{8}$. Assim, após aproximadamente dois meses, os indivíduos foram convidados a responder a um novo R24h. O retorno foi feito por telefone, obtendo-se uma taxa de resposta de $34 \%(n=294)$. A maior parte das perdas deu-se pela não localização do indivíduo, em virtude da mudança do número de telefone. No entanto, não houve diferença significativa entre a primeira e a segunda coleta em relação ao sexo e aos estratos de escolaridade do chefe da família. Ambos os R24h foram aplicados aleatoriamente, em todos os dias da semana e em todos os meses do ano. As informações contidas em cada R24h foram checadas a fim de monitorar a qualidade das entrevistas e definir a padronização para quantidades dos alimentos e receitas das preparações relatadas.

O consumo relatado foi convertido em valores de nutrientes, utilizando o software Nutrition Data System for Research (NDS, versão 2007, Nutrition Coordinating Center - NCC, University of Minnesota, Minneapolis) ${ }^{9}$. Três nutrientes foram totalizados, considerando seus equivalentes dietéticos: vitamina A (retinol somado à equivalentes de $\beta$-caroteno $)^{10}$, niacina (niacina em mg somada à quantidade convertida a partir do triptofano) ${ }^{11}$ e folato (como equivalentes dietéticos de folato) ${ }^{11}$. Foi considerada, para o ferro e o folato, a fortificação mandatória em farinhas de trigo e milho, vigente no Brasil desde 2004.

O desvio-padrão intrapessoal foi calculado utilizando modelo de efeitos aleatórios. Foi utilizada essa classe de modelos, pois eles comportam dados que violam a suposição de independência entre os mesmos indivíduos (medidas repetidas) ${ }^{12-14}$. A análise foi realizada por sexo e faixa etária, definida de acordo com o proposto pelas Dietary Recommendation Intake (DRI): adolescentes (14-18 anos), adultos (19-54 anos) e idosos (55 anos ou mais). 
O cálculo da adequação da ingestão do nutriente proposta pelo IOM foi feito segundo a equação (1):

$$
Z=\frac{\nabla-E A R}{\sqrt{\text { DPnec }+(\text { DPint } / n)}} \quad \text { (equação 1) }
$$

Na equação, $Z$ é o valor na tabela de escore- $Z$ que corresponde à probabilidade da ingestão de um dado nutriente estar adequada para um indivíduo; y é a média de ingestão de um dado nutriente obtido pela aplicação do inquérito alimentar de $n$ dias; EAR é a necessidade média estimada do nutriente; DPnec é o desvio-padrão da necessidade; e DPint é o desvio-padrão intrapessoal de consumo ${ }^{5}$.

Utilizando-se essa equação, e substituindo-se os valores de EAR e desvio-padrão das necessidades propostos pelo IOM, em conjunto com o desvio-padrão intrapessoal observado no estudo ISA-Capital, é possível estimar a quantidade adequada de nutriente $(\bar{y})$ para o indivíduo. Foram adotados três níveis de confiabilidade - 85\%, 90\% e $95 \%$ - correspondentes aos valores de $Z 1,00$, 1,28 e 1,65, respectivamente. Os valores de EAR, DPnec e Dpint foram específicos para cada nutriente, segundo sexo e faixa etária. Considerando-se que, na prática clínica, é usual solicitar o preenchimento de um registro alimentar de três dias, utilizou-se para os cálculos $n=3$.

Dessa forma, foram obtidos valores teóricos de ingestão, que podem ser comparados com o consumo relatado por um indivíduo, com o fim de averiguar a adequação de sua ingestão. Esta, por sua vez, pode ser aferida mediante a aplicação do registro alimentar de três dias, ou mediante a aplicação do R24h por três dias ${ }^{15}$.

Os dados foram analisados no pacote estatístico Stata (versão 9.2). O presente estudo foi aprovado pelo Comitê de Ética em Pesquisa da Faculdade de Saúde Pública da Universidade de São Paulo (Protocolo n 381), tendo todos os participantes assinado um Termo de Consentimento Livre e Esclarecido antes de sua inclusão na amostra.

\section{RESULTADOS}

As Tabelas 1 e 2 mostram os desvios-padrão da ingestão de nutrientes para adolescentes, adultos e idosos, de acordo com o sexo. Não foram observadas diferenças sistemáticas dos coeficientes de variação entre os sexos, exceto entre os adultos, cujos coeficientes foram menores entre os homens, na maioria dos nutrientes. Os nutrientes que apresentaram maiores discrepâncias entre as três faixas etárias em relação aos seus coeficientes de variação foram as vitaminas $A, E, K$ e C.

As Tabelas 3 e 4 mostram médias de ingestão de nutrientes que correspondem a níveis de confiabilidade de adequação de 85\%, 90\% e $95 \%$, calculados considerando o desvio-padrão intrapessoal mostrado nas Tabelas 1 e 2. Para a avaliação, compara-se o valor de ingestão do nutriente do indivíduo avaliado, obtido pela média aritmética de três dias de inquérito dietético, com os valores das tabelas, considerando o nível de confiabilidade. Valores de ingestão menores que os da tabela permitem concluir que a ingestão não está adequada para aquele nível de confiabilidade, e valores iguais ou superiores levam a concluir que a ingestão do nutriente é adequada. Como exemplo de aplicação desses valores, tome-se um indivíduo adulto, do sexo masculino, cuja média de ingestão de ferro seja de $9 \mathrm{mg}$, segundo registro alimentar de três dias. De acordo com o valor expresso na Tabela 3, conclui-se que esse indivíduo apresenta ingestão adequada de ferro, com pelo menos $95 \%$ de confiabilidade.

\section{DISCUSS ÃO}

O presente estudo disponibiliza, pela primeira vez no Brasil, valores de desvio-padrão intrapessoal da ingestão de nutrientes, necessários para obter estimativas quantitativas da adequação da dieta de um indivíduo, aplicando o método proposto pelo IOM.

Idealmente, a ingestão habitual, necessária para a avaliação da ingestão de nutrientes e ener- 
Tabela 1. Desvio-Padrão intrapessoal (DPintra) e Coeficiente de Variação (CV) da ingestão de nutrientes de acordo com o estágio da vida, no sexo masculino. São Paulo (SP), 2003.

\begin{tabular}{|c|c|c|c|c|c|c|}
\hline & \multicolumn{2}{|c|}{ Adolescente } & \multicolumn{2}{|c|}{ Adulto } & \multicolumn{2}{|c|}{ Idoso } \\
\hline & DPintra & $C V(\%)$ & DPintra & $C V(\%)$ & DPintra & $C V(\%)$ \\
\hline Vitamina A $(\mu \mathrm{g})^{\mathrm{a}}$ & 207,00 & 71 & 307,00 & 85 & 392,00 & 97 \\
\hline Vitamina E (mg) & 3,08 & 60 & 2,00 & 47 & 2,40 & 54 \\
\hline Vitamina C (mg) & 79,00 & 114 & 75,00 & 127 & 85,00 & 116 \\
\hline Tiamina (mg) & 0,70 & 48 & 0,55 & 43 & 0,45 & 43 \\
\hline Riboflavina (mg) & 0,72 & 52 & 0,70 & 45 & 0,49 & 47 \\
\hline Vitamina $B_{6}(\mathrm{mg})$ & 0,82 & 58 & 0,80 & 55 & 0,50 & 56 \\
\hline Vitamina $B_{12}(\mu \mathrm{g})$ & 2,25 & 70 & 3,30 & 73 & 2,15 & 72 \\
\hline Cálcio (mg) & 288,00 & 60 & 372,00 & 54 & 300,00 & 55 \\
\hline Fósforo (mg) & 417,00 & 45 & 582,00 & 46 & 451,00 & 50 \\
\hline Magnésio (mg) & 98,00 & 45 & 151,00 & 52 & 84,00 & 45 \\
\hline Ferro (mg) & 5,53 & 46 & 7,70 & 45 & 5,20 & 45 \\
\hline Zinco (mg) & 5,09 & 51 & 6,00 & 54 & 4,10 & 54 \\
\hline Sódio (mg) & 1540,00 & 45 & 1319,00 & 40 & 1152,00 & 51 \\
\hline Folato $(\mathrm{mg})^{\mathbf{b}}$ & 258,00 & 48 & 246,00 & 45 & 271,00 & 44 \\
\hline Niacina $(m g)^{c}$ & 15,50 & 50 & 19,80 & 51 & 9,30 & 50 \\
\hline
\end{tabular}

aexpresso em equivalentes de atividade de retinol; bexpresso em equivalente dietético de folato; cexpresso em equivalente de niacina.

Tabela 2. Desvio-Padrão intrapessoal (DPintra) e Coeficiente de Variação (CV) da ingestão de nutrientes de acordo com o estágio da vida, no sexo feminino. São Paulo (SP), 2003.

\begin{tabular}{|c|c|c|c|c|c|c|}
\hline & \multicolumn{2}{|c|}{ Adolescente } & \multicolumn{2}{|c|}{ Adulto } & \multicolumn{2}{|c|}{ Idoso } \\
\hline & DPintra & $C V(\%)$ & DPintra & $C V(\%)$ & DPintra & $C V(\%)$ \\
\hline Vitamina A $(\mu \mathrm{g})^{\mathrm{a}}$ & 223,00 & 82 & 287,00 & 73 & 375,00 & 82 \\
\hline Vitamina E (mg) & 2,16 & 59 & 2,70 & 59 & 2,07 & 50 \\
\hline Vitamina C (mg) & 59,50 & 101 & 67,00 & 122 & 96,00 & 111 \\
\hline Tiamina (mg) & 0,48 & 47 & 0,63 & 48 & 0,58 & 45 \\
\hline Riboflavina (mg) & 0,52 & 48 & 0,60 & 52 & 0,47 & 46 \\
\hline Vitamina $B_{6}(\mathrm{mg})$ & 0,68 & 61 & 0,77 & 63 & 0,72 & 53 \\
\hline Vitamina $B_{12}(\mu \mathrm{g})$ & 2,16 & 72 & 3,40 & 80 & 2,60 & 73 \\
\hline Cálcio (mg) & 315,00 & 70 & 285,00 & 60 & 259,00 & 56 \\
\hline Fósforo (mg) & 348,00 & 46 & 515,00 & 51 & 360,00 & 50 \\
\hline Magnésio (mg) & 61,50 & 45 & 95,00 & 52 & 89,00 & 46 \\
\hline Ferro (mg) & 5,21 & 49 & 5,70 & 51 & 4,50 & 48 \\
\hline Zinco (mg) & 5,23 & 59 & 5,35 & 62 & 4,70 & 49 \\
\hline Sódio (mg) & 1012,00 & 44 & 1287,00 & 50 & 1247,00 & 48 \\
\hline Folato $(\mu \mathrm{g})^{\mathbf{b}}$ & 149,00 & 43 & 270,00 & 52 & 210,00 & 43 \\
\hline Niacina $(m g)^{c}$ & 13,40 & 50 & 16,50 & 55 & 13,70 & 50 \\
\hline
\end{tabular}

aexpresso em equivalentes de atividade de retinol; bexpresso em equivalente dietético de folato; 'expresso em equivalente de niacina.

gia, deve ser obtida coletando-se vários R24h, preferencialmente em dias não consecutivos e cobrindo todos os dias da semana e todos os meses do $a_{n}{ }^{4}$. Nesse sentido, algumas publicações propõem o número de coletas necessárias para a estimativa da ingestão habitual ${ }^{16,17}$. No Brasil, Verly-Jr et al. ${ }^{3}$. estimaram o número de replicações necessárias para a avaliação de nutrientes entre adolescentes do município de São Paulo, sugerindo em torno de 15 replicações para o sexo masculino e 8 para o feminino, para apontar a maioria dos macro e micronutrientes. Entretanto, mesmo na prática clínica, tal número de dias de coleta é difícil de ser alcançado. Assim, a utilização de índices de desvio-padrão intrapessoal de ingestão, obtidos por estudos populacionais, pode ser 
830 | D.M.L. MARCHIONI et al.

Tabela 3. Valores de ingestão de nutrientesa, segundo nível de confiança, com base em registro alimentar de 3 dias, para o sexo masculino. São Paulo (SP), 2003

\begin{tabular}{|c|c|c|c|c|c|c|c|c|c|}
\hline & \multicolumn{3}{|c|}{ Adolescente } & \multicolumn{3}{|c|}{ Adulto } & \multicolumn{3}{|c|}{ Idoso } \\
\hline & $85 \%$ & $90 \%$ & $95 \%$ & $85 \%$ & $90 \%$ & $95 \%$ & $85 \%$ & $90 \%$ & $95 \%$ \\
\hline Vitamina $\mathrm{E}(\mathrm{mg})$ & 14,0 & 14,0 & 15,0 & 13,0 & 14,0 & 14,0 & 13,0 & 14,0 & 14,0 \\
\hline Tiamina (mg) & 1,5 & 1,6 & 1,8 & 1,4 & 1,6 & 1,7 & 1,4 & 1,5 & 1,7 \\
\hline Riboflavina (mg) & 1,6 & 1,7 & 1,9 & 1,6 & 1,7 & 1,9 & 1,5 & 1,6 & 1,8 \\
\hline Vitamina $B_{6}(\mathrm{mg})$ & 1,6 & 1,8 & 2,0 & 1,6 & 1,8 & 2,0 & 1,8 & 2,0 & 2,1 \\
\hline Cálcio (mg) & 1430,0 & 1467,0 & 1515,0 & 1101,0 & 1129,0 & 1166,0 & 1320,0 & 1354,0 & 1399,0 \\
\hline Fósforo (mg) & 1161,0 & 1191,0 & 1230,0 & 640,0 & 656,0 & 678,0 & 639,0 & 656,0 & 678,0 \\
\hline Magnésio (mg) & 374,0 & 384,0 & 397,0 & 364,0 & 373,0 & 386,0 & 385,0 & 395,0 & 408,0 \\
\hline Ferro $(\mathrm{mg})$ & 9,3 & 9,7 & 10,3 & 7,7 & 8,2 & 8,8 & 7,4 & 7,9 & 8,4 \\
\hline Zinco (mg) & 10,1 & 10,5 & 11,1 & 11,1 & 11,6 & 12,2 & 10,9 & 11,3 & 11,9 \\
\hline Sódio (mg) & 1652,0 & 1694,0 & 1750,0 & 1651,0 & 1694,0 & 1750,0 & 1651,0 & 1694,0 & 1750,0 \\
\hline Folato ( $\mu \mathrm{g})$ & 364,0 & 374,0 & 387,0 & 353,0 & 363,0 & 375,0 & 353,0 & 363,0 & 375,0 \\
\hline Niacina (mg) & 14,9 & 15,7 & 16,8 & 15,1 & 16,0 & 17,2 & 14,5 & 15,2 & 16,2 \\
\hline
\end{tabular}

a calculado com base na equação: $z=(\bar{y}-E A R) / \sqrt{\text { DPnec }+(\text { DPint } / n)}$

Tabela 4. Valores de ingestão de nutrientesa, segundo nível de confiança, com base em registro alimentar de 3 dias, para o sexo feminino. São Paulo (SP), 2003.

\begin{tabular}{|c|c|c|c|c|c|c|c|c|c|}
\hline & \multicolumn{3}{|c|}{ Adolescente } & \multicolumn{3}{|c|}{ Adulto } & \multicolumn{3}{|c|}{ Idoso } \\
\hline & $85 \%$ & $90 \%$ & $95 \%$ & $85 \%$ & $90 \%$ & $95 \%$ & $85 \%$ & $90 \%$ & $95 \%$ \\
\hline Vitamina E (mg) & 13,0 & 14,0 & 14,0 & 14,0 & 14,0 & 15,0 & 13,0 & 14,0 & 14,0 \\
\hline Tiamina (mg) & 1,3 & 1,4 & 1,6 & 1,4 & 1,5 & 1,7 & 1,3 & 1,5 & 1,6 \\
\hline Riboflavina (mg) & 1,3 & 1,4 & 1,6 & 1,4 & 1,5 & 1,7 & 1,3 & 1,4 & 1,6 \\
\hline Vitamina $\mathrm{B}_{6}(\mathrm{mg})$ & 1,5 & 1,6 & 1,8 & 1,6 & 1,8 & 2,0 & 1,9 & 2,1 & 2,2 \\
\hline Cálcio (mg) & 1430,0 & 1467,0 & 1515,0 & 1100,0 & 1129,0 & 1166,0 & 1320,0 & 1354,0 & 1399,0 \\
\hline Fósforo (mg) & 1161,0 & 1191,0 & 1230,0 & 639,0 & 656,0 & 678,0 & 639,0 & 656,0 & 677,0 \\
\hline Magnésio (mg) & 330,0 & 339,0 & 350,0 & 363,0 & 373,0 & 385,0 & 385,0 & 395,0 & 408,0 \\
\hline Ferro (mg) & 9,4 & 9,9 & 10,4 & 9,7 & 10,1 & 10,7 & 6,3 & 6,7 & 7,2 \\
\hline Zinco (mg) & 8,8 & 9,2 & 9,8 & 8,3 & 8,7 & 9,3 & 8,2 & 8,6 & 9,2 \\
\hline Sódio (mg) & 1651,0 & 1693,0 & 1749,0 & 1651,0 & 1694,0 & 1750,0 & 1651,0 & 1694,0 & 1750,0 \\
\hline Folato $(\mu \mathrm{g})$ & 364,0 & 373,0 & 386,0 & 353,0 & 363,0 & 375,0 & 353,0 & 362,0 & 375,0 \\
\hline Niacina (mg) & 13,7 & 14,4 & 15,4 & 13,9 & 14,7 & 15,7 & 13,7 & 14,5 & 15,4 \\
\hline
\end{tabular}

a calculado com base na equação: $z=(\bar{y}-\mathrm{EAR}) / \sqrt{\mathrm{DPnec}+(\text { DPint/n })}$.

útil na avaliação da ingestão individual, mesmo quando coletado número menor de registro alimentar ou de recordatório de 24 horas.

O uso do método proposto pelo IOM tem como mérito principal proporcionar uma estimativa quantitativa para avaliação da dieta de um indivíduo. Uma das abordagens anteriores mais utilizadas, como o cálculo percentual de consumo relativamente à ingestão dietética recomendada (Recommended Dietary Allowances - RDA), pouco adicionava à formação de um juízo de valor sobre a adequação do consumo. Ainda, traz como van- tagens o reconhecimento da variabilidade individual, tanto na ingestão, quanto nas necessidades pessoais.

No entanto, há três potenciais fontes de erro na utilização desta abordagem: (1) o coeficiente de variação de nutrientes, fixado neste estudo (10\% a 15\%), pode não representar a melhor estimativa das necessidades de ingestão; (2) a variabilidade intrapessoal de um indivíduo isolado pode ser maior ou menor do que a variância intrapessoal obtida em estudos populacionais; e (3) a presença de sub-relato de consumo pode gerar 
médias enviesadas da ingestão individual ${ }^{5}$. Considerando esses fatores, qualquer interpretação clínica a respeito da adequação de nutrientes deve ser conduzida com cautela e, de preferência, acompanhada de outros parâmetros de avaliação.

É necessário ressaltar, ainda, que esta abordagem não deve ser utilizada quando o nutriente apresente elevado desvio-padrão intrapessoal, ou seja, coeficiente de variação intrapessoal superior a $60 \%$. Na presente amostra, isso foi observado para as vitaminas $A, C$, e $B_{12}$, em todas as faixas etárias e para ambos os sexos. Nesses casos, ainda não há alternativa disponível para avaliação quantitativa da ingestão de nutrientes, considerando a metodologia recomendada pelo IOM $^{5}$.

Apesar das limitações, essa abordagem é a única recomendada pelo IOM para avaliar a adequação da ingestão de nutrientes em indivíduos. Deve-se notar, entretanto, que o simples aumento do número de dias de registro alimentar ou R24h pode gerar resultados mais acurados ${ }^{4}$.

Os valores de ingestão mostrados nas Tabelas 3 e 4 podem servir como parâmetro para avaliação da ingestão de nutrientes na prática clínica, podendo ser comparados com as médias de ingestão observadas em determinado indivíduo. Entretanto, a comparação é permitida somente quando a média de ingestão do indivíduo for proveniente de registro alimentar de 3 dias ou da aplicação de 3 recordatórios de 24 horas. Quando mais ou menos que 3 dias de coleta forem aplicados a um indivíduo, o nível de confiabilidade pode ser calculado através da equação 1. A utilização desses valores limita-se à avaliação da ingestão de nutrientes, e não deve ser usada como meta de ingestão. Para o planejamento dietético devem ser utilizados os valores de ingestão dietética recomendada (RDA)

A EAR não foi ainda estabelecida para todos os nutrientes. Nesses casos, um valor de Ingestão Adequada (Al) é a referência disponível, e, para esses nutrientes, não pode ser utilizado o método discutido acima, para estimar a adequa- ção aparente de ingestão. Chama-se a atenção para a diferença entre os valores de referência. $A$ EAR representa a mediana da necessidade do nutriente em determinado estágio de vida e sexo e, por definição, uma ingestão nesse nível pode ser inadequada para metade do grupo. Em contraste, a Al representa uma ingestão (não necessidade) que provavelmente excede a verdadeira, mas desconhecida, necessidade de quase todos os indivíduos saudáveis, dentro de um determinado sexo e estágio de vida. Quando se compara a ingestão com a Al, o que se pode concluir é se a ingestão está acima desse valor ou não. Se a ingestão habitual do nutriente estiver acima da Al, estará provavelmente adequada. Os valores de ingestão do nutriente abaixo da Al, por outro lado, ainda estarão adequados para um grupo de pessoas. No entanto, nenhuma conclusão quantitativa pode ser feita ${ }^{5}$.

Os dados não foram baseados em amostra representativa da população brasileira, mas do município de São Paulo. Ainda assim, sua utilização é considerada mais apropriada, para utilização em indivíduos brasileiros, do que valores de desvios-padrão provenientes da população norte-americana.

Por fim, a coleta do segundo R24h foi realizada por telefone, o que potencialmente poderia introduzir um viés no estudo. No entanto, foi utilizado o mesmo procedimento da coleta presencial. A primeira entrevista, presencial, pode ser considerada um momento de aprendizagem do entrevistado quanto ao método, que o habilitava a responder à segunda entrevista por telefone. Esse protocolo tem sido utilizado em estudos epidemiológicos internacionais com amostras numerosas, nos quais há cerca de 20 anos se utilizam telefone na aplicação de recordatórios de 24 horas $^{18}$, com desempenho já validado ${ }^{19}$.

\section{ONCLUSÃO}

Os desvios-padrão encontrados no presente estudo podem ser utilizados na avaliação quantitativa da adequação da ingestão de vita- 
mina $E, B_{6}$, tiamina, riboflavina, cálcio, magnésio, ferro, zinco, sódio, folato e niacina, propiciando mais um elemento para diagnóstico do estado nutricional, na avaliação clínica de indivíduos.

\section{COLABORADORES}

D.M.L. MARCHIONI responsável pelo planejamento do estudo, pela elaboração e revisão do artigo. E. VERLY JUNIOR fez as análises estatísticas e participou da elaboração e redação do artigo. C.L.G. CESAR e R.M. FISBERG participaram do planejamento do estudo e redação do artigo.

\section{REFERÊ NCIAS}

1. Fisberg RM, Marchioni DML, Slater B. Recomendações nutricionais. In: Fisberg RM, Slater B, Marchioni DML, Martini LA. Inquéritos alimentares: métodos e bases científicos. São Paulo: Manole; 2005. p.190-236.

2. Carriquiry AL. Estimation of usual intake distributions of nutrients and foods. J Nutr. 2003; 133(2):601S-08S.

3. Verly-Jr E, Cesar CLG, Fisberg RM, Marchioni DML. Sources of variation of energy and nutrient intake among adolescents in São Paulo, Brazil. Cad Saúde Pública. 2010; 26(11):2129-37. doi: 10.1590/S0 102-311X2010001100014.

4. Sempos $\mathrm{CT}$, Looker $\mathrm{AC}$, Johnson $\mathrm{CL}$, Woteki CE. The importance of withing -person variability in estimating prevalence. In: Monitoring dietary intakes. New York: Springer-Verlag; 1991. p.99-109.

5. Institute of Medicine. Food and Nutrition Board. Dietary reference intakes: applications in dietary assessment. Washington (DC): National Academy Press; 2000.

6. Willett WC. Nutritional epidemiology. $2^{\text {th }}$ ed. New York: Oxford University Press; 1998.

7. Castro MA, Barros RR, Bueno MB, César CLG, Fisberg RM. Trans fatty acid intake among the population of the city of São Paulo, Brazil. Rev Saúde Pública. 2009; 43(6):991-7. doi: 10.1590/S 0034-89102009005000084.

8. Nusser SM, Carriquiry AL, Dodd KW, Fuller WA. A semiparametric transformation Approach to
Estimating Usual Daily Intake Distributions. J Am Stat Assoc. 1996; 91(436):1440-9.

9. Nutrition Coordinating Center. Nutrition Data System for Research (NDS-R). Minnesota: University of Minnesota; 2003.

10. Institute of Medicine, Food and Nutrition Board. Dietary reference intakes for vitamin A, vitamin K, arsenic, boron, chromium, copper, iodine, iron, manganese, molybdenum, nickel, silicon, vanadium, and zinc. Washington (DC): National Academy Press; 2001.

11. Institute of Medicine. Food and Nutrition Board. Dietary reference intakes for Thiamin, Riboflavin, Niacin, Vitamin $B_{6}$, Folate, Vitamin $B_{12}$, Pantothenic Acid, Biotin, and Choline. Washington (DC): National Academy Press; 1998.

12. Verbeke G, Molenberghs G. Linear mixed models for longitudinal data. New York: Spring-Verlag; 2000. Spring Series in Statistics.

13. Littell RC, Milliken GA, Stroup WW, Wolfinger RD, Schabenberger O. SAS ${ }^{\circledR}$ for Mixed Models. $2^{\text {th }}$ ed. Cary (NC): SAS Institute; 2006.

14. Marchenko $Y$. Estimating variance components in Stata. Stata J. 2006; 6(1):1-21.

15. Marchioni DML, Slater B, Fisber RM. Aplicação das dietary reference intakes na avaliação da ingestão de nutrientes para indivíduos. Rev Nutr. 2004; 17(2):207-16. doi: 10.1590/S1415-5273200400 0200007

16. Costa MMF, Takeyama L, Voci SM, Slater B, Silva MV. Within- and between-person variations as determinant factors to calculate the number of observations to estimate usual dietary intake of adolescents. Rev Bras Epidemiol. 2008; 11(4):541-8. doi: 10.1590/S1415-790X2008000400003.

17. Jahns L, Carriquiry A, Arab L, Mroz TA, Popkin BM. Within- and between-person variation in nutrient intakes of Russian and U.S. children differs by sex and age. J Nutr. 2004; 134(11):3114-20.

18. Dwyer J, Picciano MF, Raiten DJ. Estimation of usual intake: what we eat in America - NHANES. J Nutr. 2003; 133(2):609S-3S.

19. Posner BM, Borman CL, Morgan JL, Borden WS, Ohls JC. The validity of thelephone-administered 24-hour dietary recall methodology. Am J Clin Nutr. 1982; 36(3):546-53.

Recebido em: 7/5/2010

Versão final reapresentada em: 29/6/2011 Aprovado em: 24/8/2011 